\title{
Prognostic factors in patients with advanced differentiated thyroid cancer treated with multikinase inhibitors - a single Brazilian center experience
}

\author{
Natalia Treistman ${ }^{1,2}$ \\ https://orcid.org/0000-0002-8084-5040 \\ Gabriela Maia Nobre ${ }^{1}$ \\ https://orcid.org/0000-0001-8362-1387 \\ Mariana Yoshii Tramontin' \\ https://orcid. org/0000-0002-9168-414X \\ Gabriel Madeira Werberich da Silva ${ }^{3}$ \\ https://orcid.org/0000-0003-0451-3017 \\ Daniel Herchenhorn 2,4 \\ https://orcid.org/0000-0001-5166-848X \\ Luiz Henrique de Lima Araujo ${ }^{3}$ \\ https://orcid org/0000-0001-9486-7139
}

Fernanda Accioly de Andrade ${ }^{1}$

https://orcid.org/0000-0002-6687-0506

Rossana Corbo'

https://orcid org/0000-0001-8725-7455

Daniel Bulzico'

https://orcid.org/0000-0003-1270-7241

Fernanda Vaisman ${ }^{1,2}$

https://orcid.org/0000-0002-6835-7108

\begin{abstract}
Objective: The aim of this study was to describe the real-world experience multikinase inhibitors (MKI) in the treatment advanced differentiated thyroid carcinoma (DTC) refractory to radioactive iodine (RAIR) therapy. Subjects and methods: We reviewed the records of all patients with MKItreated DTC from 2010 to 2018. Progression free survival (PFS), response rates (RR) and adverse events $(A E)$ profiles were assessed. Clinical parameters were compared between groups with different outcomes (disease progression and death) to identify possible prognostic factors and benefit from treatment. Results: Forty-four patients received MKI for progressive RAIR DTC. Median PFS was 24 months (10.2-37.7) and median overall survival (OS) was 31 months. Best overall response was complete response in one patient $(4.5 \%)$, partial response in nine $(20.4 \%)$, stable disease in twentytwo $(50 \%)$, and progressive disease (PD) in twelve (27.3\%). Seventy-two point 7 percent patients had clinical benefit and AE were mild in most cases (82.7\%). Progressive patients were more likely to have FDG positive target lesion than those who did not progress $(p=0.033)$ and higher maximum SUV on target lesions $(p=0.042)$. Presence of lung-only metastasis and lower thyroglobulin $(\mathrm{Tg})$ during treatment was associated with stable disease $(p=0.015$ and 0,049 , respectively). Patients with shorter survival had larger primary tumor size $(p=0.015)$ and higher maximum SUV on target lesions $(p=0.023)$. Conclusion: Our findings demonstrate safety and effectiveness of MKI in patients with advanced RAIR DTC. We were able to identify as possible prognostic markers of better outcomes: absence of FDG uptake on target lesions, lower maximum SUV on PET-CT, presence of lung-only metastasis and lowerTg during treatment. Arch Endocrinol Metab. 2021;65(4):411-20
\end{abstract}

Keywords

Differentiated thyroid cancer; radioactive iodine refractory; multikinase inhibitor therapy; real-world data
1 Departamento de Medicina, Serviço de Endocrinologia, Instituto Nacional do Câncer (Inca), Rio de Janeiro, RJ, Brasil 2 Departamento de Medicina, Serviço de Endocrinologia, Hospital Universitário Clementino Fraga Filho, Universidade Federal do Rio de Janeiro, Rio de Janeiro, RJ, Brasil ${ }^{3}$ Departamento de Medicina, Serviço de Oncologia, Instituto Nacional do Câncer (Inca).

Rio de Janeiro, RJ, Brasil

${ }^{4}$ Grupo de Oncologia D'Or, Instituto D'Or de Pesquisa e Educação (IDOR), Rio de Janeiro, RJ, Brasil
Correspondence to: Fernanda Vaisman

Av. Padre Leonel Franca, 110, sala 505, Gávea - 22451-000 - Rio de Janeiro, RJ Brasil

fevaisman@globo.com

Received on Nov/4/2020 Accepted on Feb/22/2021

DOI: 10.20945/2359-3997000000364 


\section{INTRODUCTION}

$\mathrm{D}$ ifferentiated thyroid carcinoma (DTC) is the most common endocrine malignancy and its incidence has been rising worldwide (1). In Brazil, estimates for 2018-2019 indicate 9610 new cases (2).

In general, DTC has excellent prognosis and over 98\% 5-year overall survival (OS) rates. Despite representing about $3 \%$ of new cancer cases in the US, it is responsible for less than $0.3 \%$ of cancer-related deaths (3). However, there is a small group of patients that can have a worse prognosis and need for additional therapy besides surgery and radioactive iodine (RAI). It is also known that patients with metastatic disease sensitive to RAI have better outcome than those who are not (4).

For patients with advanced and metastatic disease who are refractory to RAI (RAIR), therapeutic options are limited and overall response rates (RR) are also modest. Historically, it is known that DTC has poor response to cytotoxic chemotherapy $(5,6)$.

Over the last 15 years, knowledge on molecular mechanisms involved in DTC carcinogenesis and progression has evolved substantially, and with that new therapeutic possibilities were discovered (7-9). Multikinase inhibitors (MKI) were first used to treat hematologic malignancies, liver and renal cancers and were more recently approved for progressive RAIR DTC. In Brazil, the two approved MKI for RAIR DTC are sorafenib and lenvatinib, but those agents are not widely available for the public health system (10).

The experience of MKIs in DTC is still growing in many settings. Since the release of prospective controlled studies, many authors have published their experience with these agents in real-life scenarios and reported important differences in this context (11-31). However, Brazilian experience is still limited and there is no large DTC experience reported.

The aims of this study were to analyze and describe the experience of a Brazilian referral center in oncology with the use of MKI in the treatment of patients with advanced RAIR DTC and to identify predictive and prognostic factors associated with treatment.

\section{SUBJECTS AND METHODS}

We retrospectively reviewed medical records of all MKI-treated DTC patients at a single center - National Cancer Institute (Inca) -, Rio de Janeiro, Brazil, from December 2010 to November 2018.
Inclusion criteria were patients $>18$ years diagnosed with advanced DTC treated with MKI. For our analysis, we included all patients, even those with short-term treatments (less than 3 months before progression, treatment discontinuation or death).

Patients younger than 18 years old, medullary thyroid carcinoma or anaplastic thyroid carcinoma, or patients with DTC not treated with MKI were excluded.

The following demographic and clinical data from all subjects included in the analysis were collected: gender, age at diagnosis, tumor histology, number of RAI treatments, cumulative RAI activity, whole body survey (WBS) results after therapeutic RAI, criteria used to determine RAIR disease, tumor staging, metastatic lesion sites, target lesion size and site, other systemic or localized therapies performed, adequate TSH suppression prior to MKI, date and dosage of MKI treatment initiation, dosage modification when it occurred, temporary discontinuation of treatment, adverse events $(\mathrm{AE})$ and its degree when present, treatment discontinuation date and motive, anti-thyroglobulin (ATg) antibody levels and serum thyroglobulin $(\mathrm{Tg})$ before treatment, lower ATg and Tg during treatment, imaging studies during follow-up and structural response, time of last visit during followup, date of death. Tumor stage was classified according to AJCC/TNM $8^{\text {th }}$ edition (32).

Criteria used to determine RAIR disease was defined using the American Thyroid Association guidelines' definition $(6,33)$.

Patients who had clinical and radiological progressive RAIR disease were evaluated for MKI therapy. Therapy was initiated in those with symptomatic progression or with disseminated disease not manageable with localized therapy. In general, therapy was not indicated in asymptomatic patients with target lesions smaller than $2 \mathrm{~cm}$ in the largest diameter. To be eligible for treatment, patients must have had documented disease progression within 14 months.

At our institution, patients on MKI therapy are followed by a multidisciplinary team, including endocrinology, oncology, dermatology, and nurses. Depending on the case, voice therapist, clinical pain specialist and others may be involved. Initial treatment with MKI requires shorter clinical reevaluations (every 15 to 30 days) for dose adjustments and management of possible $\mathrm{AE}$, and then clinical and laboratory reassessment is performed every 2 to 3 months. The severity of $\mathrm{AE}$ is graded according to the National 
Cancer Institute Common Terminology Criteria for Adverse Events, version 4.0. Imaging and structural response studies were evaluated according to a certified radiologist (PD being defined as at least 20\% increase in measurements and partial response $[\mathrm{PR}]$ as decrease in at least $30 \%$ of target lesions).

PFS was defined as the time between initiation of MKI therapy and the first documentation of radiological disease progression, death or loss of follow-up. OS was defined as the time between MKI therapy initiation and death, loss of follow-up or last clinical visit.

Functional sensitivity of the serum Tg assay varied over the years. From 2001 to 2010, serum Tg was quantified by immunometric assay (Immulite) with functional sensitivity of $0.2 \mathrm{ng} / \mathrm{mL}$, and from 2010 to the present functional sensitivity dropped to $0.1 \mathrm{ng} / \mathrm{mL}$ (Elecsys Tg II test).

Dosages of ATg, TSH and free T4 are currently performed with electrochemiluminescence immunoassays. Functional sensitivity of ATg assay is currently $10.0 \mathrm{IU} / \mathrm{mL}$ (Elecsys Anti-Tg test). TSH Functional sensitivity is $0.005 \mu \mathrm{IU} / \mathrm{mL}$ (Elecsys TSH test) and free T4 is $0.5 \mathrm{pmol} / \mathrm{L}$ (Elecsys FT4 II test).

\section{Ethical guidelines}

This work has been approved by Inca's ethics research committee under the number 40788815.0.1001.0065.

Statistical analysis was performed using SPSS version 20.0 (SPSS Inc., Chicago, IL, USA). Continuous variables were described as means and medians, categorical variables, presented as numbers and percentages. Parametric variables were evaluated with chi-square and Student's t test. Nonparametric variables were evaluated by the Mann-Whitney U test. Survival curves were performed by the Kaplan-Meier method, and the log-rank test was used to determine statistical significance. The confidence interval is $95 \%$ and $p$ value was considered statistically significant $<0.05$.

\section{RESULTS}

In total, 44 patients were included in the analysis and their medical records were reviewed. Baseline characteristics are described in Table 1.

Twenty-seven $(61.4 \%)$ patients were female and $17(38.6 \%)$ male. Mean age at diagnosis was 60.8 and mean age at the beginning of MKI treatment 69.3 years. Regarding tumor histology, 31 patients $(70.5 \%)$ had papillary thyroid carcinoma (PTC), 12 (27.2\%) had follicular thyroid carcinoma (FTC) and l patient $(2.3 \%)$ had poorly differentiated thyroid carcinoma (PDTC). Among PTC patients, 6 had follicular variant papillary, 3 patients had insular variant, 1 tall cell $(13.6 \%, 6.8 \%$ and $2.3 \%$, respectively). Regarding FTC, 3 patients had oncotic variant $(6.8 \%)$.

Table 1. Baseline characteristics

\begin{tabular}{|c|c|c|}
\hline & $N=44$ & $\%$ \\
\hline Age (years) & $60.8(34-79)$ & \\
\hline Sex F:M & $27: 17$ & $61.4: 38.6$ \\
\hline Size $(\mathrm{cm})$ & $4.6(1.1-11.5)$ & - \\
\hline \multicolumn{3}{|l|}{ Histology } \\
\hline Papillary & 31 & 70.5 \\
\hline Follicular & 12 & 27.2 \\
\hline Poorly differentiated & 1 & 2.3 \\
\hline Follicular variant papillary & 6 & 13.6 \\
\hline Hürthle Cell & 3 & 6.8 \\
\hline Insular & 3 & 6.8 \\
\hline Tall cell & 1 & 2.3 \\
\hline \multicolumn{3}{|l|}{ 8th edition AJCC } \\
\hline Tx & 21 & 47.8 \\
\hline $\mathrm{T} 1 \mathrm{a}$ & 0 & 0 \\
\hline $\mathrm{T} 1 \mathrm{~b}$ & 0 & 0 \\
\hline T2 & 6 & 13.6 \\
\hline ТЗа & 4 & 9.0 \\
\hline T3b & 1 & 2.3 \\
\hline T4a & 7 & 15.9 \\
\hline T4b & 5 & 11.4 \\
\hline Nx & 31 & 70.5 \\
\hline NO & 1 & 2.3 \\
\hline $\mathrm{N} 1 \mathrm{a}$ & 4 & 9.0 \\
\hline N1b & 8 & 18.2 \\
\hline M1 & 23 & 52.2 \\
\hline At least one RAl treatment & 41 & 93.2 \\
\hline RAl activity (mCi) & $422.5(150-1000)$ & - \\
\hline Symptoms before MKI & 20 & 45.5 \\
\hline Time from diagnosis to MKI (years) & $68.7(0.3-210.1)$ & - \\
\hline \multicolumn{3}{|l|}{ Additional therapy besides MKI } \\
\hline External beam radiation & 27 & 61.4 \\
\hline Chemotherapy & 3 & 6.8 \\
\hline Embolization & 3 & 6.8 \\
\hline Zoledronate & 6 & 13.6 \\
\hline \multicolumn{3}{|l|}{ Final status } \\
\hline Stable disease & 11 & 25 \\
\hline Complete response & 1 & 2.3 \\
\hline Progression & 7 & 15.9 \\
\hline Disease related death & 25 & 56.8 \\
\hline PFS on MKI (months) & $24(10.2-37.7)$ & - \\
\hline OS after MKI (months) & $31(17.7-44-.2)$ & - \\
\hline Follow up (months) & $99.6(12.5-236.3)$ & - \\
\hline
\end{tabular}

MKI: multikinase inhibitors; PFS: progression free survival; OS: overall survival; RAl: radioiodine. 
Twenty-three patients (52.2\%) already had distant metastases at diagnosis. Forty-one patients were treated with RAI. They received median cumulative activity of $422.5 \mathrm{mCi}(150-1,000)$. Three patients were not treated with RAI due to unresectable disease and large remaining volume of thyroid tissue.

Criteria used to determine RAIR disease was negative WBS in 40.5\%, PD less than 16 months after RAI treatment in $27.5 \%$ and cumulative RAI activity over $600 \mathrm{mCi}$ without remission of disease in $27.5 \%$ of cases.

Regarding metastatic lesions sites, 40 patients (91\%) had pulmonary metastasis, 9 of those (20.45\%) had exclusively pulmonary metastasis. Sixteen patients $(36.3 \%)$ had bone metastasis and $10(22.7 \%)$ patients had metastasis in other sites, including liver, pancreas and the pituitary gland. Target lesions were pulmonary in 27 cases $(61.4 \%)$, cervical masses or lymph nodes in 8 cases (18.2\%), bone metastasis in 5 cases (11.4\%) and 4 cases had target lesions located in other areas. Average target lesions size was $3.1 \mathrm{~cm}$. All patients were evaluated with PET-CT, except one.

Median time between DTC diagnosis and initiation of MKI therapy was 68.7 months (0.3-210.1). Forty patients used sorafenib and 4 patients used vandetanib, all as first line treatment. Average initial dose of sorafenib was $760 \mathrm{mg} / \mathrm{d}$, with 36 patients starting 800 $\mathrm{mg}$. Initial dose for vandetanib varied between 100 $\mathrm{mg} / \mathrm{d}$ and $300 \mathrm{mg} / \mathrm{d}$.

Prior to MKI therapy $45.5 \%$ of patients had adequate TSH suppression $(\mathrm{TSH}<0.1 \mu \mathrm{IU} / \mathrm{mL}$ at least 9 of 12 previous months). Mean serum Tg before MKI was 6,469.4 $\mathrm{ng} / \mathrm{mL}$ and mean ATg titers $197.4 \mathrm{IU} / \mathrm{mL}$, mean lowest Tg during MKI treatment and lowest ATg during MKI treatment were $804.3 \mathrm{ng} / \mathrm{mL}$ and 57.9 $\mathrm{IU} / \mathrm{mL}$, respectively.

Regarding best response during treatment with MKI, 9 (20.4\%) patients had PR, 22 patients (50\%) had SD and 12 cases (27.3\%) had PD as best response during treatment as shown in Table 2. One patient presented complete response (CR) criteria and this case will be further discussed later. Overall, $72.7 \%$ patients had clinical benefit from MKI treatment, defined as the sum of CR, PR and SD. Twenty patients had symptomatic disease before starting MKI. 13 of them $(65 \%)$ reported clinical improvement of symptoms some time during treatment.

Median PFS was 24 months (10.2-37.7) (Figure 1) and median OS was 31 months (17.7-44.2)
(Figure 2). Median follow-up of 99.6 (12.5-236.3) months. Duration of response for the entire cohort was 12 months (0.5-800, for PR 12 months (8-35), for SD 31 (5-80) and for PD 9 (0-31 months).

Forty-three patients presented AE during treatment, only 1 patient had no AE reported (results in Table 3). In total, $168 \mathrm{AE}$ were described, $139(82.7 \%)$ mild

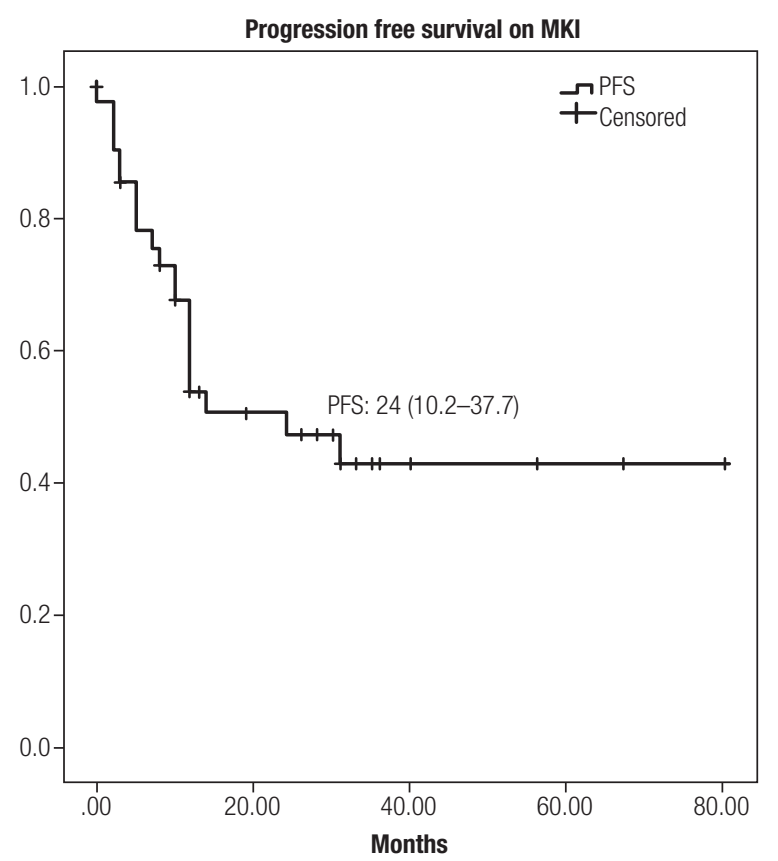

Figure 1. Progression free survival during MKI (in months).

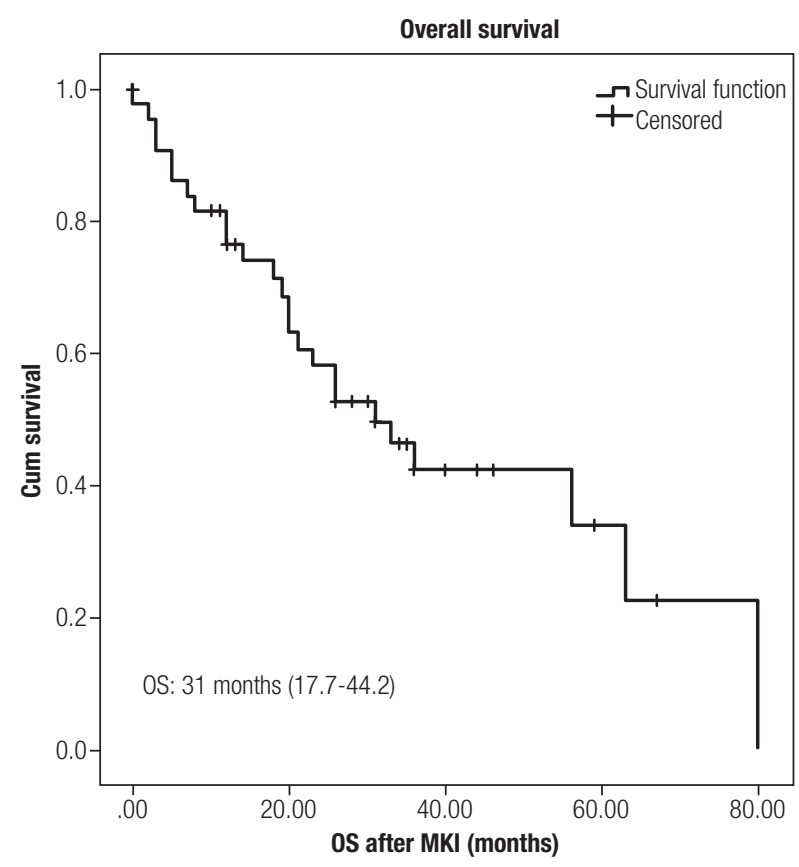

Figure 2. Overall survival after MKI (in months). 
(grades 1 or 2 ) and $29(17.3 \%)$ grade 3 or 4.21 patients $(47.7 \%)$ required temporary discontinuation of medication due to AE. Twenty-two patients (50\%) required dose reduction and 11 cases $(25 \%)$ had the drug suspended due to AE. One patient had cutaneous neoplasia secondary to MKI use.

Table 2. Response to therapy RECIST 1.1

\begin{tabular}{lc}
\hline $\mathbf{N}=\mathbf{4 4}$ & Best response to MKI therapy \\
\hline Complete response & $1(2.3 \%)$ \\
Partial response & $9(20.4 \%)$ \\
Stable disease & $22(50 \%)$ \\
Clinical benefit & $32(72.7 \%)$ \\
Disease progression & $12(27.3 \%)$ \\
\hline
\end{tabular}

MKI: multikinase inhibitors.
We also analyzed and compared data from patients who had PD on MKI with those who did not have PD while using MKI. These analyses are presented in Table 4. We found no difference between the group that progressed and those that did not progress regarding age, gender, symptoms at the beginning of MKI treatment, number of metastatic sites, number of AE or average RAI activity.

On uni-variate analysis patients who had PD on MKI were more likely to have FDG uptake on target lesions on PET-CT when compared to patients who did not progress $(\mathrm{p}=0.033)$ and higher maximum SUV on PET-CT on target lesions $(\mathrm{p}=0.042)$. Presence of lung-only metastasis was associated with no PD $(\mathrm{p}=0.02 \mathrm{l})$. Patients who did not progress had on average lower Tg during treatment when compared to

Table 3. Adverse events during MKI treatment

\begin{tabular}{lccc}
\hline & Any grade & G1-G2 (\%) & G3-G4 (\%) \\
\hline Hand-foot syndrome & 30 & $22(50)$ & $8(18.2)$ \\
Diarrhea & 31 & $25(56.8)$ & $6(13.6)$ \\
Fatigue & 31 & $29(65.9)$ & $2(4.6)$ \\
Hypertension & 5 & $4(9.0)$ & $1(2.3)$ \\
Alopecia & 11 & $9(20.4)$ & $3(6.8)$ \\
Anorexia & 5 & $5(11.3)$ & 0 \\
Weight loss & 8 & $8(18.2)$ & 0 \\
Nausea & 9 & $8(18.2)$ & $1(2.3)$ \\
Rash & 7 & $5(11.3)$ & $2(4.6)$ \\
Hematologic toxicity & 1 & $1(2.3)$ & 0 \\
Pruritus & 1 & $1(2.3)$ & 0 \\
Secondary neoplasia & 1 & 0 & $1(2.3)$ \\
\hline
\end{tabular}

MKI: multikinase inhibitors.

Table 4. Progression on MKI

\begin{tabular}{lccc}
\hline & Progression (22) & No progression (22) & p-value \\
\hline Age (years) & 59 & 66 & 0.561 \\
Sex (F) & $50 \%$ & $72.7 \%$ & 0.215 \\
Primary tumor size (cm) & 4.5 & 3.7 & 0.057 \\
Number of metastatic sites & & & 0.03 \\
1 & $13.6 \%$ & $45.5 \%$ & $31,8 \%$ \\
2 & $45.5 \%$ & $18.2 \%$ & $4.5 \%$ \\
3 & $22.7 \%$ & $36.36 \%$ & $\mathbf{0 . 0 2}$ \\
4 & $18.2 \%$ & 11.0 & $\mathbf{0 . 0 4 2}$ \\
Pulmonary metastasis only & $4.5 \%$ & $85.7 \%$ & $\mathbf{0 . 0 3 3}$ \\
Max. SUV - PET + Target lesion & 14.62 & 165.5 & $\mathbf{0 . 0 4 9}$ \\
PET + Target lesion & $100 \%$ & $36.4 \%$ & 0.364 \\
Sowest Tg during MKI & 664.9 & 4.0 & 0.213 \\
Numbtomatic disease & $54.5 \%$ & 473.81 & 0.057 \\
Mean RAl activity & 3.0 & & \\
\hline
\end{tabular}

MKI: multikinase inhibitors; Tg: thyroglobulin; RAl: radioiodine, AE: adverse events. 
patients who progressed $(\mathrm{p}=0.049)$, however there was no statistically significant correlation with initial $\mathrm{Tg}$.

We performed analysis comparing patients who died during or after MKI treatment and survivors, as shown in Table 5.

Patients who died had larger primary tumor size $(\mathrm{p}=0.035)$, more frequently had more than one site of distant metastasis $(\mathrm{p}=0.002)$ and higher incidence of glucose uptake on target lesions on PET-CT $(\mathrm{p}=0.023)$.

\section{DISCUSSION}

In this study we describe a retrospective cohort of patients with progressive unresectable DTC RAIR, treated with MKI for a median period of 99.6 months in a public referral center in Rio de Janeiro. This larger Brazilian experience showed that, in a real-world study, median PFS was 24 months (10.2-37.7) and OS was 31 months (17.7-44.2), with frequent but manageable adverse events in properly selected patients.

Despite the favorable results of previous phase III studies, there are still many unresolved questions regarding the clinical management of patients treated with MKI treated RAIR DTC. Chief among them is how such results are converted to a real-life scenario practice. Several groups have begun to describe their experience with treating DTC using MKI and its feasibility in many different countries, continents, and contexts $(13-31,34,35)$. Findings of previous colleagues as well as our results are summarized in Table 6. Our study represents a large single center cohort treated with MKI, with long follow-up, being one of the few cohorts in South American and the first with Brazilian population.

Regarding survival outcomes, our findings are slightly different from previous phase III studies but consistent with other groups reports of real-world experience, such as Cabanillas and cols. with 19 months PFS in a North American cohort, Benekli and cols. with 21.3 months PFS in Turkish population, Molina-Vega and cols. with 18 months PFS in a Spanish cohort, Sugino and cols. 24.3 months in a Japanese cohort, and Jerkovich and cols. with 31.5 months PFS in an Argentinian cohort (11-13,18,25,26,29).

Clinical trials DECISION and SELECT have previously showed PFS of 10.8 months and 18.3 months, respectively, an improvement when compared to their placebo groups, respectively, 5.8 and 3.6 months $(11,12)$. Although not directly comparable, considering all our subjects presented documented PD within 14 months prior to MKI initiation, we believe our finding of median 24 months PFS demonstrates the usefulness of MKI treatment to prevent disease progression.

Most of our patients experienced clinical benefit of treatment. $50 \%$ of them had SD, 20.4\% PR and one presented CR. This patient was started on MKI after presenting a rapidly progressive unresectable endotracheal lesion that can no longer be seen on cross sectional images after 28 months of sorafenib. Our $72.7 \%$ clinical benefit was similar to Marotta and cols.

Table 5. Disease related death

\begin{tabular}{lccc}
\hline & Deaths (25) & Survivors (19) & p-value \\
\hline Age & 61.16 & 60.42 & 0.649 \\
Sex (F) & $72 \%$ & $47.4 \%$ & 0.125 \\
Primary tumor size & 5.47 & 3.6 & $\mathbf{0 . 0 3 5}$ \\
Number of metastatic sites & & & $\mathbf{0 . 0 2}$ \\
1 & $20 \%$ & $42.2 \%$ & \\
2 & $44 \%$ & $31.5 \%$ & \\
3 & $16 \%$ & $26.3 \%$ & 0.023 \\
4 & $20 \%$ & $84.2 \%$ & 0.088 \\
PET + target lesion & $96 \%$ & 701.75 & 0.135 \\
Lowest Tg during MKI & 914.11 & $31.6 \%$ & 0.689 \\
Symptomatic disease & $56 \%$ & $75 \%$ & 0.530 \\
PD target lesion vs. Non target lesion & $64.7 \%$ & 4.1 & 0.407 \\
Number of AE & 3.6 & 386.11 & \\
Mean RAl activity & 452.27 & & \\
\hline
\end{tabular}

MKl: multikinase inhibitors; Tg: thyroglobulin; RAl: radioiodine, AE: adverse events. 
Table 6. Review of world real-life experience in use of MKI in DTC

\begin{tabular}{|c|c|c|c|c|c|c|c|c|}
\hline Country & Year & Authors & $\begin{array}{l}\text { Number of } \\
\text { centers }\end{array}$ & Drugs & $\begin{array}{l}\text { Number of } \\
\text { subjects }\end{array}$ & $\begin{array}{l}\text { 1st line } \\
\text { MKI or } \\
\text { more }\end{array}$ & Median PFS (months) & Prognostic Factors \\
\hline $\begin{array}{l}\text { United } \\
\text { States }\end{array}$ & 2010 & $\begin{array}{l}\text { Cabanillas } \\
\text { and cols. }\end{array}$ & Single center & $\begin{array}{l}\text { Sorafenib } \\
\text { Sunitinib }\end{array}$ & 15 DTC & $\begin{array}{l}1^{\text {st }} \text { line or } \\
\text { more }\end{array}$ & 19 & $\begin{array}{l}\text { Yes: } \\
\text { Log Tg }\end{array}$ \\
\hline Italy & 2013 & $\begin{array}{l}\text { Marotta } \\
\text { and cols. }\end{array}$ & Single center & Sorafenib & 17 & $1^{\text {st }}$ line & 9 & $\begin{array}{l}\text { Yes: } \\
\text { Tg levels and Tg response to } \\
\text { treatment, baseline FDG-PET }\end{array}$ \\
\hline France & 2014 & $\begin{array}{l}\text { Massicotte } \\
\text { and cols. }\end{array}$ & Multicenter & $\begin{array}{l}\text { Sorafenib } \\
\text { Sunitinib } \\
\text { Vandetanib }\end{array}$ & $\begin{array}{c}45 \text { DTC } \\
\text { (17 MTC) }\end{array}$ & $\begin{array}{l}1^{\text {st }} \text { line or } \\
\text { more }\end{array}$ & 7.0 (1st line DTC) & No \\
\hline Turkey & 2015 & $\begin{array}{l}\text { Benekli and } \\
\text { cols. }\end{array}$ & $\begin{array}{l}\text { Unclear (Turkish } \\
\text { Ministry of } \\
\text { Health } \\
\text { database) }\end{array}$ & Sorafenib & $\begin{array}{c}14 \text { DTC } \\
\text { (16 MTC) }\end{array}$ & Unclear & 21.3 (DTC group) & No \\
\hline France & 2017 & $\begin{array}{l}\text { Berdelou } \\
\text { and cols. }\end{array}$ & Multicenter & Lenvatinib & 75 & $\begin{array}{l}1^{\text {st line or }} \\
\text { more }\end{array}$ & 10 & No \\
\hline Spain & 2018 & $\begin{array}{l}\text { Molina- } \\
\text { Vega and } \\
\text { cols. }\end{array}$ & Single center & $\begin{array}{l}\text { Sorafenib } \\
\text { Lenvatinib } \\
\text { Axitinib }\end{array}$ & 17 & $\begin{array}{l}1^{\text {st }} \text { line or } \\
\text { more }\end{array}$ & 18 & No \\
\hline Korea & 2018 & $\begin{array}{l}\text { Mijin Kim } \\
\text { and cols. }\end{array}$ & Multicenter & Sorafenib & 98 & $1^{\text {st }}$ line & 9.7 & $\begin{array}{c}\text { Yes: } \\
\text { Symptoms, lung-only } \\
\text { metastasis, daily maintenance } \\
\text { dose, Tg reduction }\end{array}$ \\
\hline Switzerland* & 2018 & $\begin{array}{l}\text { Balmelli } \\
\text { and cols. }\end{array}$ & Multicenter & Lenvatinib & 13 & $\begin{array}{l}1^{\text {st line or }} \\
\text { more }\end{array}$ & 7.2 & $\begin{array}{c}\text { Yes: Tg levels (with radiologic } \\
\text { response) }\end{array}$ \\
\hline Japan & 2018 & Sugino & Single center & Lenvatinib & 29 & $\begin{array}{l}1^{\text {st line or }} \\
\text { more }\end{array}$ & 24.3 & Symptom \\
\hline Korea & 2019 & $\begin{array}{l}\text { Kim and } \\
\text { cols. }\end{array}$ & Multicenter & Sorafenib & 85 & $\begin{array}{l}1^{\text {st }} \text { line or } \\
\text { more }\end{array}$ & 14.4 & $\begin{array}{c}\text { Yes: } \\
\text { Small tumor size, long doubling } \\
\text { time }\end{array}$ \\
\hline Japan & 2019 & $\begin{array}{l}\text { Suzuki and } \\
\text { cols. }\end{array}$ & Single center & Lenvatinib & 26 & $\begin{array}{l}1^{\text {st line or }} \\
\text { more }\end{array}$ & 2 year-PFS $=58.4 \%$ & $\begin{array}{c}\text { Yes: } \\
\text { Baseline tumor size and } \\
\text { symptoms }\end{array}$ \\
\hline Japan & 2019 & $\begin{array}{l}\text { Yamazaki } \\
\text { and cols. }\end{array}$ & Single center & Lenvatinib & 36 & $\begin{array}{l}1^{\text {st }} \text { line or } \\
\text { more }\end{array}$ & $\begin{array}{l}\text { Full Dose: } 696 \text { days } \\
\text { Low Dose: not reached }\end{array}$ & No \\
\hline Korea & 2019 & $\begin{array}{l}\text { Lee and } \\
\text { cols. }\end{array}$ & Multicenter (11) & Lenvatinib & 67 & $\begin{array}{l}1^{\text {st line or }} \\
\text { more }\end{array}$ & 5.1 & $\begin{array}{l}\text { Yes: } \\
\text { Rapidly PD with shorter initial } \\
\text { tumor doubling time }\end{array}$ \\
\hline Italy & 2019 & $\begin{array}{l}\text { Locati and } \\
\text { cols. }\end{array}$ & Multicenter (16) & Lenvatinib & 94 & $\begin{array}{l}1^{\text {st line or }} \\
\text { more }\end{array}$ & 10.8 & No \\
\hline Argentina & 2019 & $\begin{array}{l}\text { Jerkovich } \\
\text { and cols. }\end{array}$ & Single center & $\begin{array}{l}\text { Sorafenib } \\
\text { Lenvatinib }\end{array}$ & 22 & $\begin{array}{l}1^{\text {st }} \text { line or } \\
\text { more }\end{array}$ & $\begin{array}{c}31.5 \\
\left(16.5-1^{\text {st l line only) }}\right.\end{array}$ & No \\
\hline Japan & 2019 & $\begin{array}{l}\text { Iwasaki and } \\
\text { cols. }\end{array}$ & Multicenter & $\begin{array}{l}\text { Sorafenib } \\
\text { Lenvatinib }\end{array}$ & 56 & $1^{\text {st }}$ line & $\begin{array}{c}\text { Median treatment } \\
\text { duration: } \\
\text { Sorafenib 5.1 Lenvatinib } \\
14.1\end{array}$ & $\begin{array}{c}\text { Yes: } \\
\text { Pulmonary metastasis as target } \\
\text { lesion }\end{array}$ \\
\hline Portugal & 2019 & $\begin{array}{l}\text { Santos and } \\
\text { cols. }\end{array}$ & Single center & $\begin{array}{l}\text { Sorafenib } \\
\text { Sunitinib }\end{array}$ & 28 & $\begin{array}{l}1^{\text {st }} \text { line or } \\
\text { more }\end{array}$ & 10.8 (1st line sorafenib) & No \\
\hline China & 2020 & $\begin{array}{l}\text { Cheng and } \\
\text { cols. }\end{array}$ & Single center & Sorafenib & 72 & $1^{\text {st }}$ line & 17.6 & $\begin{array}{c}\text { Yes: } \\
\text { Hand-foot syndrome, } \\
\text { Well DTC, ECOG PS } \leq 2 \text {, } \\
\text { biochemically nonineffective } \\
\text { response, lung-only metastasis, } \\
\text { and absence of bone metastasis }\end{array}$ \\
\hline
\end{tabular}




\begin{tabular}{|c|c|c|c|c|c|c|c|c|}
\hline Country & Year & Authors & $\begin{array}{l}\text { Number of } \\
\text { centers }\end{array}$ & Drugs & $\begin{array}{l}\text { Number of } \\
\text { subjects }\end{array}$ & $\begin{array}{l}\text { 1st line } \\
\text { MKI or } \\
\text { more }\end{array}$ & Median PFS (months) & Prognostic Factors \\
\hline Argentina & 2020 & $\begin{array}{l}\text { Jerkovich } \\
\text { and cols. }\end{array}$ & Multicenter (02) & Lenvatinib & 22 & $\begin{array}{l}1^{\text {st line or }} \\
\text { more }\end{array}$ & 13.7 & No \\
\hline Netherlands & 2020 & $\begin{array}{l}\text { Aydermirli } \\
\text { and cols. }\end{array}$ & Multicenter (03) & Lenvatinib & 39 & $\begin{array}{l}1^{\text {st }} \text { line or } \\
\text { more }\end{array}$ & 9.7 & No \\
\hline Japan & 2020 & $\begin{array}{l}\text { Masaki and } \\
\text { cols. }\end{array}$ & Single center & Lenvatinib & 42 & $\begin{array}{l}1^{\text {st }} \text { line or } \\
\text { more }\end{array}$ & 13.8 & No \\
\hline Brazil & 2020 & $\begin{array}{l}\text { Treistman } \\
\text { and cols. }\end{array}$ & Single center & Sorafenib & 44 & $1^{\text {st }}$ line & 24 & $\begin{array}{c}\text { FDG uptake on target lesions on } \\
\text { PET-CT, higher SUV presence of } \\
\text { lung-only metastasis and lower } \\
\text { Tg during treatment }\end{array}$ \\
\hline
\end{tabular}

TTg: thyroglobulin; DTC: differentiated thyroid cancer; MTC: medullary thyroid cancer; PFS: progression free survival; OS: overall survival; RAI: radioiodine.

71\% (30\% PR and 41\% SD) and Iwasaki and cols.'s $75.0 \%$ disease control rate (PR plus SD) $(14,34)$.

Three of our patients did not receive RAI due to unresectable disease and large remaining volume of thyroid tissue, similar cases have also been reported in previous cohorts. Santos and cols., Berdelou and cols. as well as Locati and cols. also described in each report patients that did not undergo thyroid surgery before starting MKI therapy due to unresectable tumors $(16,22,30)$. Those patients would not be eligible for previous MKI trials, however in our experience, two of those three patients had clinical benefit of MKI treatment (one PR and one SD).

When we compared groups divided by outcomes (PD on MKI versus no PD) we found no difference regarding age, number of $\mathrm{AE}$ or average RAI activity. We also found no difference regarding symptoms at the beginning of MKI treatment and disease progression as some groups have previously reported. Both Suzuki and cols. and Sugino and cols. have reported that tumorrelated symptom were prognostic factors for both poorer PFS and OS in Japanese cohorts $(29,36)$. Kim and cols. also found such association in a multicenter Korean cohort (20). This difference in our results could be explained due to sample size or perhaps different studied population. Even though symptomatic disease did not correlate with PD or death outcomes in our study, $65 \%$ of patients who had symptomatic disease before starting MKI reported clinical improvement of symptoms some time during treatment. Berdelou and cols. also described that $52 \%$ of their 44 patients with initial symptoms related to DTC had clinical improvement of symptoms (16).

Another interesting finding was that presence of lung-only metastasis was associated with no PD $(\mathrm{p}=$
0.021 ) and that patients who did not progress had on average lower Tg during treatment when compared to patients who progressed $(\mathrm{p}=0.049)$. Kim and cols. also described association between lung-only metastasis and PFS, Cheng and cols. also reported that better PFS and OS were found in patients with lung-only metastasis $(17,20)$.

Several authors also found correlations between $\mathrm{Tg}$ levels and response to MKI. First, Cabanillas and cols. reported that lower $\log \mathrm{Tg}$ was associated to better radiological response (13). Marotta and cols. described that baseline $\mathrm{Tg}$ levels were significantly higher in patients who showed disease progression, as well as correlation between baseline Tg and PFS (14). This group also reported that the decrease in serum $\mathrm{Tg}$ levels was significantly greater in patients who achieved clinical benefit. In Balmelli and cols.'s report decrease in Tg levels correlated with radiologic response in 6 evaluated patients (31). In Korean population, 60\% $\mathrm{Tg}$ reduction was associated with better PFS, and more recently Cheng and cols. biochemically response (decrease $\mathrm{Tg}$, stable $\mathrm{Tg}$ or increases of under $25 \%$ ) independently predicted PFS and OS $(17,20)$.

As of major interest, risk factors for cancer-specific mortality was deeply explored. Our group found no difference regarding age, gender, PD site, number of AEs, symptoms at the beginning of MKI therapy or mean RAI activity. Patients who died had larger primary tumor size $(p=0.035)$ and higher incidence of glucose uptake on target lesions on PET-CT $(\mathrm{p}=0.023)$. Our group also showed that patients who evolved with $\mathrm{PD}$ had a higher incidence of FDG uptake on target lesions on PET-CT when compared to patients who did not progress $(\mathrm{p}=0.033)$ and higher maximum SUV on PETCT on target lesions $(\mathrm{p}=0.042)$. Association between 
PET-CT findings and response to MKI treatment in RAIR DTC patients is in line with previous reports by Marotta and cols. (14). In their work, baseline average SUVmax was significantly higher in patients who showed disease progression compared with responding subjects, however no significant correlation with PFS was found. Kim and cols. more recently described that the presence of FDG-PET uptake did not affect PFS in his cohort (21). We believe the use of PET-CT in MKI treated RAIR DTC patients should be further analyzed in larger cohorts since we found it as useful in clinical practice.

Regarding safety, most patients presented side effects during MKI treatment. Similar to previous trials, the majority of $\mathrm{AE}$ were low grade $(11,12)$. However, in $50 \%$ of cases, reducing medication dosage was necessary at some point to manage side effects, similarly to Santos and cols. and Balmelli and cols. $(30,31)$. In $25 \%$ of cases the drug was eventually suspended due to AE, also reported by Kim and cols.'s (23\% permanent discontinuation) - but higher than reported by Jerkovich and cols. and Benekli and cols. with only 1 patient in each series permanently suspending sorafenib $(18,21,25)$. Only one secondary cutaneous neoplasia was found in our cohort. Squamous cell carcinoma was found in 7 out of 207 sorafenib treated patients in DECISION trial, and other colleagues reported similar occurrences $(11,13,18)$. The fact that almost every patient will experience $\mathrm{AE}$ at one point during $\mathrm{MKI}$ treatment and that $\mathrm{AE}$ might interfere with ongoing treatment highlights the importance of an experienced assistant team to manage such drugs.

Our work, however, has limitations. As a retrospective cohort, we had some cases of loss of follow-up. In addition, when we perform chart analysis, we have come across some missing data. The limited size of our sample may limit conclusions and reduce statistical power. As any study in a real-life setting, there are often difficulties in scheduling and performing exams, poor adherence to treatment, missed appointments and other factors that may interfere in some way with the results.

Nevertheless, is the first Brazilian report and one of the few subcontinental cohorts validating findings in other populations and demonstrating safety and efficacy of the use of MKI in RAIR-DTC. Our findings also corroborate previous authors that found presence of lung-only metastasis, absence of FDG uptake on target lesions on PET-CT, lower maximum SUV on PET-CT and lower $\mathrm{Tg}$ during treatment associated with better outcomes in RAIR DTC patients treated with MKI.

In conclusion, our analysis demonstrates that the use of MKI drugs in patients with advanced RAIR DTC is a safe and effective therapeutic approach and results were consistent with international literature data, with median PFS of 24 months (10.2-37.7) and 72.7\% clinical benefit from MKI treatment. We were able to identify absence of FDG uptake on target lesions on PET-CT, lower maximum SUV on PET-CT, presence of lung-only metastasis and lower $\mathrm{Tg}$ during treatment as possible prognostic markers.

Disclosure: no potential conflict of interest relevant to this article was reported.

\section{REFERENCES}

1. Lim H, Devesa SS, Sosa JA, Check D, Kitahara CM. Trends in Thyroid Cancer Incidence and Mortality in the United States, 1974-2013. JAMA. 2017;317(13):1338-48.

2. Instituto Nacional de Câncer José Alencar Gomes da Silva (Inca). Estimativa 2018: incidência de câncer no Brasil. Rio de Janeiro: Inca; 2017.

3. National Institutes of Health (NIH). SEER Cancer Statistics Review, 1975-2015. 2018. Available from: https://seer.cancer.gov/ archive/csr/1975_2015/

4. Durante C, Haddy N, Baudin E, Leboulleux S, Hartl D, Travagli JP, et al. Long-term outcome of 444 patients with distant metastases from papillary and follicular thyroid carcinoma: benefits and limits of radioiodine therapy. J Clin Endocrinol Metab. 2006;91(8): 2892-9.

5. Sherman SI. Cytotoxic chemotherapy for differentiated thyroid carcinoma. Clin Oncol (R Coll Radiol). 2010;22(6):464-8.

6. Haugen BR, Alexander EK, Bible KC, Doherty GM, Mandel SJ, Nikiforov YE, et al. 2015 American Thyroid Association Management Guidelines for Adult Patients with Thyroid Nodules and Differentiated Thyroid Cancer: The American Thyroid Association Guidelines Task Force on Thyroid Nodules and Differentiated Thyroid Cancer. Thyroid. 2016;26(1):1-133.

7. Berdelou A, Lamartina L, Klain M, Leboulleux S, Schlumberger $M$. Treatment of refractory thyroid cancer. Endocr Relat Cancer. 2018;25(4):R209-R223.

8. Durante C, Tallini G, Puxeddu E, Sponziello M, Moretti S, Ligorio $\mathrm{C}$, et al. BRAF(V600E) mutation and expression of proangiogenic molecular markers in papillary thyroid carcinomas. Eur J Endocrinol. 2011;165(3):455-63.

9. Bible KC, Ryder M. Evolving molecularly targeted therapies for advanced-stage thyroid cancers. Nat Rev Clin Oncol. 2016;13(7):403-16.

10. Agência Nacional de Vigilância Sanitária (Anvisa). Homepage: http://portal.anvisa.gov.br/.

11. Brose MS, Nutting CM, Jarzab B, Elisei R, Siena S, Bastholt $L$, et al. Sorafenib in radioactive iodine-refractory, locally advanced or metastatic differentiated thyroid cancer: a randomised, doubleblind, phase 3 trial. Lancet. 2014;384(9940):319-28.

12. Schlumberger M, Tahara M, Wirth LJ, Robinson B, Brose MS, Elisei $R$, et al. Lenvatinib versus placebo in radioiodine-refractory thyroid cancer. N Engl J Med. 2015;372(7):621-30. 
13. Cabanillas ME, Waguespack SG, Bronstein Y, Williams MD, Feng L, Hernandez M, et al. Treatment with tyrosine kinase inhibitors for patients with differentiated thyroid cancer: the M. D. Anderson experience. J Clin Endocrinol Metab. 2010;95(6):2588-95.

14. Marotta V, Ramundo V, Camera L, Del Prete M, Fonti R, Esposito R, et al. Sorafenib in advanced iodine-refractory differentiated thyroid cancer: efficacy, safety and exploratory analysis of role of serum thyroglobulin and FDG-PET. Clin Endocrinol (Oxf). 2013;78(5):760-7.

15. Aydemirli MD, Kapiteijn E, Ferrier KRM, Ottevanger PB, Links TP, van der Horst-Schrivers ANA, et al. Effectiveness and toxicity of lenvatinib in refractory thyroid cancer: Dutch real-life data. Eur J Endocrinol. 2020;182(2):131-8.

16. Berdelou A, Borget I, Godbert $Y$, Nguyen T, Garcia ME, Chougnet $\mathrm{CN}$, et al. Lenvatinib for the Treatment of Radioiodine-Refractory Thyroid Cancer in Real-Life Practice. Thyroid. 2018;28(1):72-8.

17. Cheng L, Fu H, Jin Y, Sa R, Chen L. Clinicopathological Features Predict Outcomes in Patients with Radioiodine-Refractory Differentiated Thyroid Cancer Treated with Sorafenib: A RealWorld Study. Oncologist. 2020;25(4):e668-78.

18. Jerkovich F, García Falcone MG, Pitoia F. The experience of an Endocrinology Division on the use of tyrosine multikinase inhibitor therapy in patients with radioiodine-resistant differentiated thyroid cancer. Endocrine. 2019;64(3):632-8.

19. Jerkovich F, Califano I, Bueno F, Carrera JM, Giglio R, Abelleira E, et al. Real-life use of lenvatinib in patients with differentiated thyroid cancer: experience from Argentina. Endocrine. 2020;69(1):142-8.

20. Kim M, Kim TH, Shin DY, Lim DJ, Kim EY, Kim WB, et al. Tertiary Care Experience of Sorafenib in the Treatment of Progressive Radioiodine-Refractory Differentiated Thyroid Carcinoma: A Korean Multicenter Study. Thyroid. 2018;28(3):340-8.

21. Kim MJ, Kim SM, Lee EK, HwangboY, LeeYJ, Cho SW, et al.Tumor doubling time predicts response to sorafenib in radioactive iodine-refractory differentiated thyroid cancer. Endocr J. 2019;66(7):597-604.

22. Locati LD, Piovesan A, Durante C, Bregni M, Castagna MG, Zovato S, et al. Real-world efficacy and safety of lenvatinib: data from a compassionate use in the treatment of radioactive iodinerefractory differentiated thyroid cancer patients in Italy. Eur J Cancer. 2019;118:35-40.

23. Masaki C, Sugino K, Saito N, Akaishi J, Hames KY, Tomoda C, et al. Efficacy and Limitations of LenvatinibTherapy for RadioiodineRefractory DifferentiatedThyroid Cancer: Real-World Experiences. Thyroid. 2020;30(2):214-21.

24. Nervo A, Gallo M, Samà MT, Felicetti F, Alfano M, Migliore $E$, et al. Lenvatinib in Advanced Radioiodine-refractory Thyroid Cancer: A Snapshot of Real-life Clinical Practice. Anticancer Res. 2018;38(3):1643-9.

25. Benekli M, Yalcin S, Ozkan M, Elkiran ET, Sevinc A, Cabuk D, et al. Efficacy of sorafenib in advanced differentiated and medullary thyroid cancer: experience in a Turkish population. Onco Targets Ther. 2014;8:1-5.

26. Molina-Vega M, García-Alemán J, Sebastián-Ochoa A, ManchaDoblas I, Trigo-Pérez JM, Tinahones-Madueño F. Tyrosine kinase inhibitors in iodine-refractory differentiated thyroid cancer: experience in clinical practice. Endocrine. 2018;59(2):395-401.

27. Massicotte $\mathrm{MH}$, Brassard $M$, Claude-Desroches $M$, Borget I, Bonichon F, Giraudet $A L$, et al. Tyrosine kinase inhibitor treatments in patients with metastatic thyroid carcinomas: a retrospective study of the TUTHYREF network. Eur J Endocrinol. 2014;170(4):575-82.

28. Lee EK, Kim SM, Kim BH, Kim MJ, Lim DJ, Kim MH, et al. LesionBased Evaluation Predicts Treatment Response to Lenvatinib for Radioactive lodine-Refractory Differentiated Thyroid Cancer: A Korean Multicenter Retrospective Study. Thyroid. 2019;29(12):1811-9.

29. Sugino K, Nagahama M, Kitagawa W, Ohkuwa K, Uruno T, Matsuzu K, et al. Clinical factors related to the efficacy of tyrosine kinase inhibitor therapy in radioactive iodine refractory recurrent differentiated thyroid cancer patients. Endocr J. 2018;65(3): 299-306.

30. Sousa Santos F, Joana Santos R, Leite V. Sorafenib and Sunitinib for theTreatment of MetastaticThyroid Cancer of Follicular Origin: A 7-Year Single-Centre Experience. EurThyroid J. 2019;8(5):262-7.

31. Balmelli C, Railic N, Siano M, Feuerlein K, Cathomas R, Cristina $\mathrm{V}$, et al. Lenvatinib in Advanced Radioiodine-Refractory Thyroid Cancer - A Retrospective Analysis of the Swiss Lenvatinib Named Patient Program. J Cancer. 2018;9(2):250-5.

32. Tuttle RM, Haugen B, Perrier ND. Updated American Joint Committee on Cancer/Tumor-Node-Metastasis Staging System for Differentiated and Anaplastic Thyroid Cancer (Eighth Edition): What Changed and Why? Thyroid. 2017;27(6):751-6.

33. Vaisman F, Carvalho DP, Vaisman M. A new appraisal of iodine refractory thyroid cancer. Endocr Relat Cancer. 2015;22(6): R301-10.

34. Iwasaki H, Yamazaki H, Takasaki H, Suganuma N, Sakai R, Nakayama $\mathrm{H}$, et al. Treatment outcomes of differentiated thyroid cancer with distant metastasis improve by tyrosine kinase inhibitors. Oncol Lett. 2019;17(6):5292-300.

35. Yamazaki H, Iwasaki H, Takasaki H, Suganuma N, Sakai R, Masudo $\mathrm{K}$, et al. Efficacy and tolerability of initial low-dose lenvatinib to treat differentiated thyroid cancer. Medicine (Baltimore). 2019;98(10):e14774.

36. Suzuki C, Kiyota N, Imamura Y, Goto H, Suto H, Chayahara N, et al. Exploratory analysis of prognostic factors for lenvatinib in radioiodine-refractory differentiated thyroid cancer. Head Neck. 2019;41(9):3023-32. 